\title{
OTIMIZAÇÃO DO PROCESSO DE DESTILAÇÃO EM PLANTA PILOTO PARA PRODUÇÃO DE BIOETANOL HIDRATADO
}

\author{
L. T. SCHNEIDER ${ }^{1}$, G. BONASSA ${ }^{1}$, C. de OLIVEIRA ${ }^{1}$, J. T. TELEKEN ${ }^{2}$, P. A. \\ CREMONEZ $^{3}$, J. G. TELEKEN ${ }^{1}$, \\ ${ }^{1}$ Universidade Federal do Paraná - Setor Palotina \\ ${ }^{2}$ Universidade Federal de Santa Catarina, Departamento de Engenharia Química e \\ Engenharia de Alimentos \\ ${ }^{3}$ Universidade Estadual do Oeste do Paraná \\ E-mail para contato: laarats@gmail.com
}

\begin{abstract}
RESUMO - No processo de destilação a separação dos componentes líquidos miscíveis é feita através da diferença de volatilização entre eles. Obtendo as variáveis que proporcionam maior rendimento e menor gasto energético, o objetivo do trabalho é a otimização da produção de etanol fixando valores de: tempo de produção de etanol dentro da especificação de álcool hidratado, taxa de refluxo e vazão volumétrica de produto de topo. Realizaram-se seis processos de destilação em sistema descontínuo, com a alteração de três variáveis independentes: razão de refluxo, pressão de vapor do refervedor e porcentagem de álcool na mistura a ser destilada, e a partir disto, avaliou-se a influência dessas variáveis de entrada do processo sobre as variáveis respostas: tempo de start-up, volume de etanol produzido, vazão (L.h-1) de etanol e graduação alcoólica do produto. Durante os experimentos monitoraram-se os perfis de temperatura do destilador, alambique, condensador e deflegmador, para avaliar como o comportamento destes podem afetar o processo de destilação. Após a realização dos testes, analisando o menor tempo como objetivo principal do processo, tem se o experimento 2 como o de maior rendimento, que durou aproximadamente 74 minutos com uma vazão de 43,9 L.h-1 e um total de produto retirado acima de $92{ }^{\circ} \mathrm{GL}$ de 16 litros. Neste teste utilizou-se uma taxa de refluxo de 0,75 , pressão de vapor de $0,7 \mathrm{Kgf} / \mathrm{cm}^{-2}$ e graduação alcoólica inicial de mistura de $11 \% \mathrm{~V} / \mathrm{V}$.
\end{abstract}

\section{INTRODUÇÃO}

A destilação é um processo de separação amplamente utilizado na indústria química, com grande aplicação nas áreas petroquímicas e dos biocombustíveis, e que consiste na evaporação parcial da mistura líquida de acordo com a diferença de pontos de ebulição/volatilização, tendo como objetivo principal a separação de determinado composto do volume da mistura, agregando valor ao produto final. Valor que depende exclusivamente da sua pureza e qualidade, tornando a condução e o controle do processo essências para 
alcançar a máxima produção, qualidade e rentabilidade (Parissoto et al., 2009).

Dentre as etapas de produção do etanol a destilação é considerada a mais onerosa, atingindo até $80 \%$ do custo operacional industrial por demandar grande fluxo energético, geralmente se apresentando como a etapa de maior limitância (Teixeira, 2003). E justamente por possuir um custo energético muito elevado há a preocupação quanto à sua otimização.

Colunas de destilação requerem o emprego de sistemas de controle e métodos operacionais ajustados, rejeitando flutuações que ocorrem frequentemente, até mesmo quando o processo está operando em fase estacionária. $\mathrm{O}$ comportamento não-linear das variáveis, associado às elevadas constantes de tempo, restrições na operação, atrasos na resposta e diferentes volumes processados, invariavelmente afetam o processo resultando em acréscimos significativos nos custos operacionais, grande volume produzido fora das especificações desejadas e prejudicando a obtenção de maiores eficiências (Marangoni et al., 2007).

A pressão de vapor, a taxa de refluxo e a graduação alcoólica inicial da mistura são variáveis que afetam diretamente o processo de destilação e a qualidade do produto de topo.

A taxa de refluxo é definida como a razão de líquido advindo do condensador que retorna à coluna, ou seja, utiliza-se parte do destilado na forma de refluxo fazendo com que os componentes menos voláteis sejam retidos dos vapores que ascendem pela coluna, aumentando a concentração de leves no topo e consequentemente a pureza do destilado (Cadore, 2011). O refluxo ótimo deve ser seguido de acordo com um critério ótimo de desempenho: produção máxima dentro de especificação em tempo mínimo, tempo mínimo para produção de determinada quantidade de produto dentro de especificação, ou lucro máximo (Fileti, 1995).

A partir deste cenário, o objetivo do presente trabalho foi avaliar a produtividade de etanol em unidade piloto, utilizando diferentes parâmetros operacionais a fim de aperfeiçoar a produção e o consumo energético, tomando como índices de desempenho: o tempo necessário para produção de etanol dentro da especificação de álcool hidrato; a taxa de refluxo necessária; e a vazão volumétrica de produção do etanol.

\section{MATERIAIS E MÉTODOS}

Para que os sistemas operacionais sejam executados e mantidos deve-se fazer o monitoramento de determinados fatores que influenciam no processo, ajustando-os e controlando-os. Os fatores variados durante os testes foram: fração volumétrica de etanol, taxa de refluxo e pressão de vapor.

\subsection{Fração volumétrica de etanol}

A mistura a ser destilada possuía aproximadamente 300 litros água/álcool, e realizouse a correção da graduação alcoólica através do cálculo de diluição (Equação 1), de acordo com os valores de porcentagens alcoólicas estabelecidas, especificados em: 5\%, 8\% e $11 \%$. 


$$
C_{1 .} V_{1}=C_{2 .} V_{2}
$$

(Equação 1)

Onde: $\mathrm{C}_{1}$ : Concentração alcoólica inicial; $\mathrm{V}_{1}$ : Volume inicial; $\mathrm{C}_{2}$ : Concentração desejada; $\mathrm{V}_{2}$ : Volume desejado.

\subsection{Refluxo}

A taxa de refluxo é um fator determinante no teor alcoólico do destilado. Os valores testados foram de: $0,25,0,5$ e 0,75 , regulados na válvula de refluxo contida no painel interno do sistema de destilação.

\subsection{Pressão de vapor}

Após o aquecimento da caldeira, quando esta atingir aproximadamente $5 \mathrm{Kgf.cm}{ }^{-2} \mathrm{de}$ vapor, abre-se a válvula que permite a liberação de pressão para a estrutura de destilação. Utilizou-se três diferentes valores do fator pressão de vapor: $0,35,0,70$ e $1,05 \mathrm{Kgf} / \mathrm{cm}^{-2}$, observados no manômetro contido no painel interno da estrutura de destilação.

Na Tabela 1, observam-se os valores de taxa de refluxo, graduação alcoólica inicial e pressão de vapor utilizados em cada experimento.

Tabela 1. Planejamento experimental

\begin{tabular}{cccc}
\hline Número de Ensaios & \%Etanol (v/v) & $\begin{array}{c}\text { Valores Reais } \\
\text { Refluxo }\end{array}$ & $\begin{array}{c}\text { Pressão de vapor } \\
\left(\text { Kgf.cm }^{-2}\right)\end{array}$ \\
\hline 1 & 5 & 0,5 & 0,7 \\
2 & 11 & 0,5 & 0,7 \\
3 & 8 & 0,25 & 0,7 \\
4 & 8 & 0,75 & 0,7 \\
5 & 8 & 0,5 & 0,35 \\
6 & 8 & 0,5 & 1,05 \\
\hline
\end{tabular}

\subsection{Vazão volumétrica do destilado $\left({\left.\mathrm{L} . \mathrm{h}^{-1}\right)}^{-1}\right.$}

Realizou-se o cálculo da vazão em cada um dos testes após a retirada do destilado com graduação alcoólica acima de $92^{\circ} \mathrm{GL}$, através da equação 2 . 


$$
Q=V / t
$$

(Equação 2)

Onde: Q: Vazão; V: Volume retirado; t: Tempo.

\section{RESULTADOS E DISCUSSÃO}

Com base nos dados da Tabela abaixo, observa-se a influência das diferentes condições de operação das variáveis reais sobre o tempo do processo, volume de etanol produzido, vazão de produto de topo e graduação alcoólica do produto retirado.

Tabela 2 - Valores reais e variáveis respostas dos experimentos

\begin{tabular}{cccccccc}
\hline $\begin{array}{c}\text { Número } \\
\text { de Ensaios }\end{array}$ & \% Etanol & Refluxo & Pressão & $\begin{array}{c}\text { Tempo } \\
(\text { min) }\end{array}$ & $\begin{array}{c}\text { Variáveis Respostas } \\
\text { etanol } \\
(\mathbf{L})\end{array}$ & $\begin{array}{c}\text { Vazão } \\
(\mathbf{L} / \mathbf{h})\end{array}$ & ${ }^{\mathbf{o}} \mathbf{G L}$ \\
\hline 1 & 5 & 0,5 & 0,7 & 40 & 0 & 0 & 90 \\
2 & 11 & 0,5 & 0,7 & 74 & 16 & 43,9 & 92 \\
3 & 8 & 0,25 & 0,7 & 140 & 14 & 18,9 & 92 \\
4 & 8 & 0,75 & 0,7 & 165 & 16 & 13,0 & 92 \\
5 & 8 & 0,5 & 0,35 & 298 & 36 & 7,5 & 92 \\
6 & 8 & 0,5 & 1,05 & 42 & 0 & 0 & 91 \\
\hline
\end{tabular}

\subsection{Perfis de temperatura dos equipamentos de destilação}

Durante os processos realizados ocorreu o monitoramento das temperaturas do equipamento de destilação, com o objetivo de analisar a influência destas, relacionando-as com a composição e vazão do produto de topo.

Os perfis de temperaturas podem ser observados na Figura 1. 

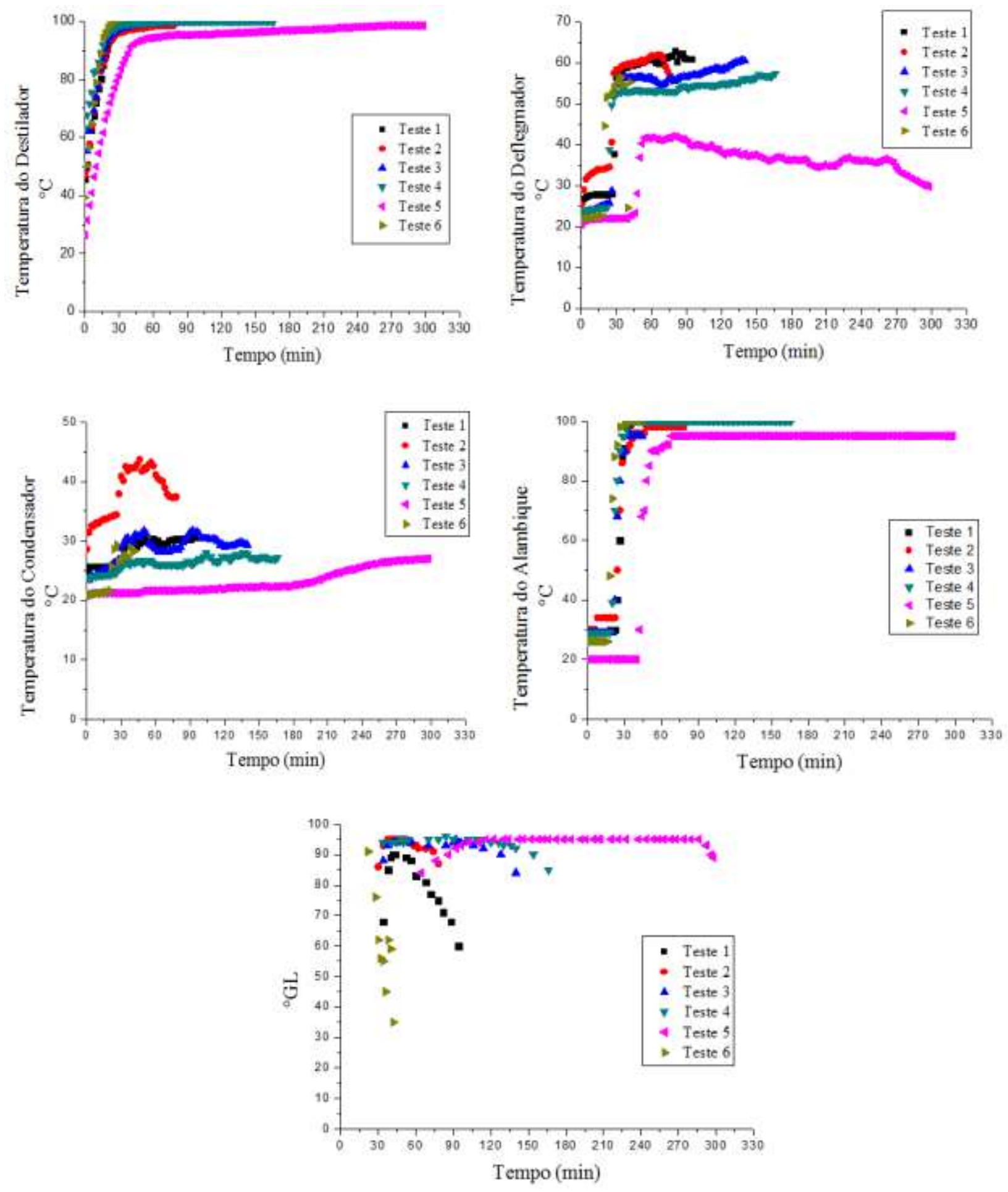

Figura 1 - Perfis de temperatura do processo de destilação e graduação alcoólica do produto retirado

Observa-se na Figura 1 que as temperaturas do destilador seguiram o mesmo comportamento durante todos os testes, aumentando gradativamente até atingir uma temperatura próxima de $100{ }^{\circ} \mathrm{C}$, mantendo esta estabilidade até o fim do processo, e o mesmo padrão de aquecimento manteve-se também para as temperaturas do alambique. Por todos os componentes da mistura à ser destilada possuírem ponto de volatilização até 100 
${ }^{\circ} \mathrm{C}$, todos os componentes da mistura são destilados sem que temperaturas maiores desses equipamentos precisem ser atingidas.

A temperatura do condensador é medida através da sua água de resfriamento. Quanto maior a fração de água presente nestes vapores que chegam até este equipamento, maior a troca de calor necessária para que haja a condensação dessa mistura, resultando em maiores temperaturas. Na Figura 1, observa-se que durante o experimento 5, a temperatura do condensador manteve-se entre 20 e $27{ }^{\circ} \mathrm{C}$, indicando uma elevada fração de álcool na mistura devido a baixa temperatura requerida para a condensação dos vapores.

O deflegmador tem como função produzir um refluxo interno, responsável por enriquecer a graduação alcoólica do produto de topo. Quando os perfis de temperatura do deflegmador se mantêm em uma temperatura menor do que a do ponto de bolha da mistura, este consegue reter o vapor que chega até ele, produzindo um refluxo interno e conduzindo os vapores ao condensador com uma alta graduação alcoólica.

Enquanto misturas de mesma composição são condensadas e chegam ao defllegmador, a temperatura deste permanece estável e há a retirada de produto com graduação alcoólica de interesse. Quando há alteração na composição desta mistura devido a mudanças da pressão de vapor ou algum outro fator, a temperatura do deflegmador também é afetada, geralmente elevada, devido ao aumento da fração de água na mistura.

Em relação à graduação alcoólica do produto de topo, observa-se que inicialmente o produto é retirado com uma baixa graduação, devido à ausência de refluxo e a não estabilidade das temperaturas. Posteriormente, a graduação aumenta e permanece estável durante o período de start-up, diminuindo novamente quando há alteração na pressão de vapor ou diminuição da graduação alcoólica da mistura a ser destilada..

\subsection{Análise das variáreis respostas}

A maioria dos processos industriais busca a redução dos tempos de operação, diminuindo assim seus gastos energéticos (Werle et al., 2007). A partir do teste 6, observase que o tempo de destilação é reduzido de forma significativa com a utilização de uma maior pressão de vapor. Entretanto, o produto retirado não está dentro das especificações em relação ao ${ }^{\circ} \mathrm{GL}$, não sendo de interesse comercial. Além disso, há um gasto energético muito elevado, portanto, as condições não se mostraram vantajosas em relação à obtenção de produto, e devem ser otimizadas para que haja maior eficiência no processo.

De acordo com os resultados obtidos, observa-se que a pressão de vapor exerce influencia direta na condução do processo de destilação, determinando um maior ou menor tempo nos testes realizados. A utilização de maiores pressões de vapor faz com que a mistura a ser destilada seja aquecida rapidamente, produzindo maior pressão interna e uma maior quantidade de vapores que ascendem pela coluna. Devido a essa velocidade da fase vapor, os fenômenos de transferência de calor que e massa entre as fases líquido/vapor não são suficientes para que o vapor seja enriquecido, resultando na retirada de produto de baixa graduação alcoólica, como observado no teste 6 . Já os testes realizados com uma menor pressão de vapor apresentaram melhores resultados em relação à retirada de produto dentro da especificação, porem, em maiores tempos. 
O volume de etanol produzido está diretamente relacionado com a porcentagem alcoólica inicial da mistura, onde todo o volume de álcool adicionado na mistura inicial deve ser destilado, portanto quanto maior este volume, maior a quantidade produzida. Este produto retirado pode ou não possui alta graduação alcoólica, e quanto menor a graduação do produto, o volume produzido tende a ser maior, pois o este possui também certa quantidade de água em sua composição.

A taxa de refluxo e a pressão de vapor também podem influenciar positiva ou negativamente neste volume. Observa-se que no teste 2 , mesmo possuindo um maior valor de porcentagem alcoólica inicial do que o teste 5, retirou-se uma menor quantidade de produto desejado, devido a utilização de diferentes pressões de vapor em cada um destes testes, onde, a utilização de pressão de vapor mais elevada provoca o rápido aquecimento da mistura a ser destilada, gerando um maior fluxo de vapores na coluna, o que dificulta os fenômenos de troca de calor e massa entre a fase líquido/vapor, e faz com que o produto condensado possua baixa graduação alcoólica.

Realizou-se o cálculo da vazão apenas durante os processos que atingiram a graduação alcoólica desejada (acima de $92{ }^{\circ} \mathrm{GL}$ ), determinando assim o tempo aproximado de retirada do álcool adicionado na mistura a ser destilada de acordo com a porcentagem alcoólica de cada experimento.

A maior vazão foi atingida no teste 2 , cerca de 43,9 L.h. ${ }^{-1}$, operado com uma taxa de refluxo 0,5 , pressão de vapor de $0,7 \mathrm{Kgf.cm}^{-2}$ e porcentagem alcoólica inicial de $11 \% \mathrm{~V} / \mathrm{V}$. E o valor de vazão mínimo foi do teste 5 , cerca de $7,5 \mathrm{~L} \cdot \mathrm{h}^{-1}$, que utilizou uma taxa de refluxo de 0,5 , pressão de vapor de $0,35 \mathrm{Kgf.cm}^{-2}$ e porcentagem alcoólica inicial de $8 \% \mathrm{~V} / \mathrm{V}$. O que pode ter influenciado na baixa vazão de produto de topo do teste 5 é a menor pressão de vapor, o que causou o mais lento aquecimento da coluna, aumentando o tempo de start-up, de retirada de produto e o tempo final do processo.

Para a comercialização do produto do processo de destilação, este deve passar por uma série de análises de controle de qualidade e estar dentro dos parâmetros de certificação da ANP (Agência Nacional do Petróleo, Gás natural e Biocombustíveis). Dentre essas especificações está a graduação alcoólica, que no caso do álcool hidratado deve estar acima de $92{ }^{\circ} \mathrm{GL}$.

Nem todos os testes atingiram este valor, devido às condições de operação fornecidas nos processos. Os fatores que podem ter influenciado nessa variável resposta são: baixa graduação alcoólica inicial da mistura, como no caso do teste 1 , onde a fração de álcool na mistura é muito baixa, fazendo com que o produto de topo não possua a graduação alcoólica desejada nessas condições de pressão de vapor e refluxo; e elevada pressão de vapor, como no exemplo do teste 6 , onde esta, como explicado anteriormente, dificulta o aumento da concentração alcoólica dos vapores devido a elevada velocidade em que estes passam pela coluna de destilação.

\section{CONCLUSÃO}

Após a realização dos experimentos, a obtenção dos resultados e a análise destes, concluiu-se que a pressão de vapor exerce grande influencia sobre o tempo dos processos e o volume de etanol retirado, pois esta diretamente ligada ao tempo de aquecimento da mistura 
do sistema de destilação e a velocidade em que os vapores formados ascendem pela coluna, onde quanto maior a pressão de vapor, menor o tempo de start-up, e maior a velocidade da fase vapor.

Para a determinação do melhor teste é necessário que o objetivo final do processo seja definido, por exemplo, tempo, volume de etanol produzido ou graduação alcoólica do retirado.

Analisando o menor tempo como objetivo principal do processo, tem se o experimento 2 como o de maior rendimento, que durou cerca de 74 minutos, com uma vazão de 43,9 L.h ${ }^{-1}$ e um total de produto retirado acima de $92{ }^{\circ} \mathrm{GL}$ de 16 litros. Neste teste utilizou-se uma taxa de refluxo de 0,75 , pressão de vapor de $0,7 \mathrm{Kgf.cm}{ }^{-2}$ e graduação alcoólica inicial de mistura de $11 \%$ v/v.

Devido à alta taxa de refluxo houve a purificação da fase vapor, e a retirada de produto com a graduação alcoólica desejada. A pressão de vapor, por ser alta, também pode ter influenciado em um menor tempo de start-up da coluna, diminuindo o tempo deste, e aumentando o tempo da fase estacionária (fase em que as temperaturas do sistema de destilação não sofrem grande variação, e que o produto de interesse é produzido).

\section{REFERENCIAS}

CADORE, I. R. Eficiência de torres de destilação. 2011.46 f. Tese de Graduação em Engenharia Química. Universidade Federal do Rio Grande do Sul, Porto Alegre. 2011.

FILETI, A. M. F. Controle em destilação batelada: controle adaptativo e controle preditivo com modelo baseado em redes neurais artificiais. $216 \mathrm{f}$. Tese de Doutorado em Engenharia Química. Universidade Estadual de Campinas, Campinas. 1995.

MARANGONI, C., LEANDRO OSMAR WERLE, L.O., MACHADO, R. A. F. Controle multivariável com ação distribuída em uma coluna de destilação. $4^{o}$ PDPETRO, Campinas-SP, 2007.

PARISOTTO, I. G. B., OLIVEIRA, D. L., WERLE, L. O., MARANGONI, C., MACHADO, R. A. F. Uso de software comercial para estudo das variáveis da fase vapor de um processo de destilação quando utilizada uma nova estratégia de controle. $5^{o}$ Congresso brasileiro de pesquisa e desenvolvimento em petróleo e gás. Fortaleza-CE, 2009.

TEIXEIRA, A. C. Inferências em Coluna de Destilação Multicomponente. Monografia. Curso de Graduação em Engenharia Química. UFSC, Florianópolis-SC, 2003.

WERLE, L. O., MARANGONI, C., STEINMACHER, F. R., ARAÚJO, P. H.H. DE., MACHADO, R. A. F., SAYER, C. Estudo da etapa de aquecimento na partida de uma coluna de destilação comparando os efeitos da ação distribuida de calor com a convencional. $4^{\circ}$ PDPETRO, Campinas-SP, 2007. 